\title{
Development and validation of the Czech version of the Athletic Coping Skills Inventory (ACSI-28) for soccer referees
}

\author{
Michal Vičar ${ }^{1, *}$, Vít Koukal², Václav Šašinka ${ }^{3}$, and Pavlína Vaculíková2 \\ ${ }^{1}$ Faculty of Physical Culture, Palacký University Olomouc, Olomouc, Czech Republic; ${ }^{2}$ Faculty of Sports Studies, Masaryk \\ University, Brno, Czech Republic; and ${ }^{3}$ Faculty of Social Studies, Masaryk University, Brno, Czech Republic
}

Copyright: (c) 2020 M. Vičar et al. This is an open access article licensed under the Creative Commons Attribution License (https://creativecommons.org/licenses/by/4.0/).

Background: Athletic Coping Skills Inventory (ACSI-28) is a widely used questionnaire in sport psychology for athletes. It has been previously adapted into several languages. However, not only athletes but also the referees must resist the enormous pressure and cope with stress. Objective: This paper aims to create the Czech version of the questionnaire with the target population of soccer referees, and formulate recommendations for the future development of the tool both in original and adapted versions. Methods: The questionnaire was distributed to 199 referees from the target population, predominantly males $(94.5 \%)$ of performance levels ranging from regional to international. The process of translation and back-translation was carried out. The reliability, validity, and factor structure of the Czech version of this inventory was analysed and compared with the original and Spanish versions. Results: The factor structure of the Czech version of ACSI- 28 was assessed satisfactory (comparative fit index $=.85$, Tucker-Lewis index $=.87$, root mean square error of approximation $=.052$, standardized root mean square residual $=.072$ ). The reliability in the form of internal consistency was confirmed in most of the subscales (varying from .78 to .62). The subscale Coachability showed higher internal consistency (.65) when one question was not included. The subscale of Confidence and Achievement Motivation showed low internal consistency (.55). The subscale Freedom from Worry should not be included in the overall Coping Resources scale. After excluding the Freedom from Worry subscale, the tool displays good reliability (.87). Conclusions: The Czech version of ACSI-28 for soccer referees includes 27 items. With the consideration of its limits, the Czech version of ACSI-28 can be used in practice as a screening method for soccer referees.

Keywords: coping, soccer referees, scale standardization, mental skills

\section{Introduction}

The effect of the psychological factors on sports performance is a frequently researched phenomenon (e.g., MacNamara, Button, \& Collins, 2010; Orlick, 1992; Vičar, 2018). Usually, these mental characteristics associated with top-level sports are referred to as mental skills (Lesyk, 1998). Conversely, the mental skills and mental preparation in referees are much less popular topic, with the soccer referees being no exception.

It is a shared belief that the pressure that soccer referees face is enormous, and the impact of their decisions is far-reaching, not only in tense matches. This

\footnotetext{
* Address for correspondence: Michal Vičar, Department of Recreation and Leisure Studies, Faculty of Physical Culture, Palacký University Olomouc, třída Míru 117, 77111 Olomouc, Czech Republic. E-mail: michal.vicar@upol.cz
}

places great demands on their coping level - the ability to manage stress. The requirement for high quality coping in soccer referees is emphasised by many studies (Anshel \& Weinberg, 1995; Gencay, 2009; Mathers \& Brodie, 2011). For example, it seems that in the face of a stressful event, strategies of avoidance rather than approach are usually used by soccer referees (Salehi \& Mohseni, 2018). Coping strategies are also described to play an important role in referee successful long-term career development (Samuel, Galily, \& Tenenbaum, 2017). At the same time, it is believed that the increasing level of referees leads to a higher level of their coping strategies with higher anxiety levels (Johansen \& Haugen, 2013). Experiencing stress connected with the fear of failure of bad decision shows the potential to influence the depression rate (Voborný, Zeman, Blahutková, \& Beňuš, 2015). Therefore, concerns about the mental health of referees are being 
raised (Mellick, 2020). Yet, in addition to the Referee Self-Efficacy Scale (Myers, Feltz, Guillén, \& Dithurbide, 2012), only a few specialized methods assess referees' psychological profile. It opens the space for creating new methods. Apart from developing the new ones, the second option is the adaptation of the existing methods to the population of referees from related fields - sports area.

The level of mental skills in sports - especially those associated with coping is assessed by the Athletic Coping Skill Inventory - 28 (ACSI-28) scale (Smith, Schutz, Smoll, \& Ptacek, 1995). This tool is one of the most widespread questionnaires used by sports psychologists worldwide, for example, the scale was translated from English to Spanish (Sanz, Pérez, Coll, \& Smith, 2011) and Greek (Goudas, Theodorakis, $\&$ Karamousalidis, 1998). Yet the adaptation for the Czech athletes has not been created. As the original ACSI-28 formulations are rather universal, it possibly enables their application to soccer referees and even to the general population of referees in related sports fields. Therefore we believe, that after minor modifications, ACSI-28 might be a suitable instrument for diagnostics of coping skills among soccer referees.

This study aims to create ACSI-28 questionnaire adapted for soccer referees in the Czech language. It requires not only translation of the original questionnaire but also reformulation of some items for the new population, followed by assessing the reliability and validity of the Czech version upon the target population. Based on the findings, the recommendation for the future development of the Czech version of the questionnaire for referees but also the original version of the questionnaire for athletes will be formulated.

\section{Methods}

\section{Participants}

The research sample consisted of 199 respondents, soccer referees from various competition levels in the Czech Republic and internationally. The respondents were divided into two groups. One group comprised international and national referees $(n=58)$; the other group included regional referees $(n=141$, including referees from regional and district leagues). At the same time, all respondents were holders of a valid licence and were registered with the Football Association of the Czech Republic. The length of a professional referee experience was between 1 and 35 years $(M=9.1$, $S D=6.27)$. For more details, see Table 1.

The questionnaire was distributed electronically. The respondents were contacted by email. The sample was established using purposive sampling. Before completing the questionnaire, the respondents signed an informed consent form. Data anonymity was ensured.

\section{Research instrument}

The Athletic Coping Skills Inventory-28 (ACSI-28) is the third version of this questionnaire. The questionnaire is based on a theoretical model that utilizes information about stress, social support and coping with mental stress. The first, version of the questionnaire, entitled "Survey of Athletic Experience" contained 87 items in 8 subscales. Using the factor analysis, the number of items was reduced to 42 for the second version. Based on the low psychometric properties and in-adequate factor structure, another 14 items and one subscale were excluded. Consequently, at present, the ACSI-28 has 28 items ( 7 subscales, each containing 4 questions). The questions are formulated as statements with a 4-point Likert response scale (almost never, sometimes, often, and almost always). The questionnaire also includes basic demographic information such as age, gender, performance level, and length of sports experience. The ACSI-28 includes the following subscales (listed with operationalized definitions; Kimbrough, DeBolt, \& Balkin, 2007):

1. Coping with Adversity: Respondents achieving a high score in this category remain positive and enthusiastic even in the case of failure, etc. They have complicated situations under control and mostly solve them in a calm way without emotions. They also have the ability to recover and learn from their own mistakes and setbacks quickly.

2. Peaking under Pressure: Respondents achieving a high score in this subscale have learned to work under pressure and are able to perform well in

Table 1

Descriptive characteristics of the Czech soccer referees sample

\begin{tabular}{|c|c|c|c|c|c|c|c|}
\hline \multirow[b]{2}{*}{ Level } & \multicolumn{3}{|c|}{ Gender } & \multicolumn{4}{|c|}{ Age (years) } \\
\hline & Male & Female & Total & $M$ & $S D$ & Minimum & Maximum \\
\hline Regional & 133 & 8 & 141 & 27.51 & 8.68 & 16 & 55 \\
\hline National/international & 55 & 3 & 58 & 29.19 & 6.19 & 20 & 50 \\
\hline Total & 188 & 11 & 199 & 28.01 & 8.05 & 16 & 55 \\
\hline
\end{tabular}


complicated situations. Under pressure, they show higher commitment and effort.

3. Goal Setting/Mental Preparation: Respondents achieving a high score in this area responsibly prepare for a performance and are able to set and define specific performance targets. They have a predetermined plan to perform well.

4. Concentration: Respondents achieving a high score in this area maintain concentration in all sorts of adverse situations. They have the capacity to fully concentrate on a specific task even under difficult and extreme conditions.

5. Freedom from Worry: Respondents achieving a high score in this area do not worry about unfavourable situations and mistakes that might occur in a match. They do not subject themselves to unnecessary pressure. They do not worry about other people's criticism concerning their mistakes and do not needlessly focus on negative situations that they want to avoid.

6. Confidence and Achievement Motivation: A high score in this area suggests the ability to work hard to improve their skills, be positively motivated, believe in their abilities, and push the boundaries of their capabilities.

7. Coachability: Respondents achieving a high score in this subscale have the ability to accept criticism and learn from it. It is usually easy for them to learn new things, and they are open to comments and instructions.

All the subscales combined create Coping Resources total sum score of the questionnaire. To develop the Czech version of the ACSI-28, the process of translation and back-translation was used (three translators to Czech, one to English). The process was performed according to the instructions presented by Banville, Desrosiers, and Genet-Volet (2000), and in particular Vallerand (1989). The recommendations formulated by the International Testing Commission were also respected during the translation, back-translation, and discussion phases. One of the reasons why the ACSI28 questionnaire was chosen for adaptation was the universality of the formulations of the items. Based on the face validity, the items were suitable even for the population of the soccer referees. Still, some items were slightly adapted (Nos. 3, 4, 6, 9, 10, 15, and 27) to focus specifically on soccer referees. However, the semantic core of all items remained identical, including their meaning in the context of coping strategies. The adequacy and comprehensibility of the new formulations of items were agreed by the expert committee composed of three people involved in the field (sports psychologist, soccer referee, and professional translator
- linguist). The process of adaptation was run with the consent of the authors of the questionnaire.

\section{Statistical data processing}

The data from the Czech sample were analysed using SPSS Statistics (Version 22.0; IBM, Armonk, NY, USA). Item analysis was performed. Inter item correlations below .20 were considered weak correlations, $.20-.25$ borderline correlations, and .25-.60 ideal range correlations, as higher values may indicate that the items may only be capturing a small bandwidth of the construct. For item-total correlation with the scale, we used .30 as a satisfactory level for keeping the item in the scale. Cronbach's alpha of individual subscales and stratified Cronbach's alpha was calculated for the entire questionnaire. We used the following values for evaluating reliability: $\geq .90$ for excellent reliability, $.80-.90$ for good reliability, $.70-.80$ for acceptable reliability, .60-.70 for questionable reliability, $.50-.60$ for poor reliability, and $<.50$ for unacceptable reliability (P. Kline, 1993).

Confirmatory factor analysis (CFA) was performed using the robust maximum likelihood approach using the following indexes. The comparative fit index (CFI) and Tucker-Lewis index (TLI) compare a specified model with a "baseline" model based on a hypothesis that the items are not correlated. In an ideal case, their values should be close to 1 . Other indicators used in the analysis were the root mean square error of approximation (RMSEA) and standardized root mean square residual (SRMR). R. B. Kline (2015) suggests that the top value of its $90 \%$ confidence interval should not exceed.10. Scale unidimensionality was tested by Horn's parallel analysis.

To assess the criterion validity of the method, Student's $t$-test was used to measure the differences between national and international referees forming first group, and regional referees forming the second group. Cohen's $d$ was used to assess the effect size. The differences between performance levels were assessed as follows: small effect (0.2-0.5), medium effect (0.5$0.8)$, and large effect (>0.8).

Regarding the low number of women in the research sample (5.6\%), which is due to the overall low number of Czech female referees, only values for the entire research sample are specified.

\section{Results}

\section{Item analysis}

Table 2 shows the correlation matrix of the items for each subscale. The items are identified using abbreviations. In all items, the corrected correlations with the 
Table 2

Results of inter-item correlations of subscales and descriptive values for Czech soccer referees sample

\begin{tabular}{|c|c|c|c|c|c|c|}
\hline & \multicolumn{3}{|c|}{ Inter-item correlation } & \multirow[b]{2}{*}{$M$} & \multirow[b]{2}{*}{$S D$} & \multirow[b]{2}{*}{$\alpha ?$} \\
\hline & 1 & 2 & 3 & & & \\
\hline \multicolumn{7}{|c|}{ Coping with Adversity } \\
\hline Cop1 & - & & & 1.75 & 0.81 & .56 \\
\hline Cop2 & .35 & - & & 1.75 & 0.86 & .54 \\
\hline Cop3 & .38 & .35 & - & 1.49 & 0.83 & .54 \\
\hline Cop4 & $.20 *$ & .27 & .27 & 2.05 & 0.80 & .63 \\
\hline \multicolumn{7}{|c|}{ Peaking under Pressure } \\
\hline Pea 1 & - & & & 1.65 & 0.86 & .72 \\
\hline Pea2 & .52 & - & & 1.75 & 0.97 & .68 \\
\hline Pea3 & .39 & .56 & - & 1.80 & 0.93 & .74 \\
\hline Pea4 & .51 & .46 & .35 & 1.78 & 0.83 & .74 \\
\hline \multicolumn{7}{|c|}{ Goal Setting/Preparation } \\
\hline GS1 & - & & & 1.38 & 0.86 & .60 \\
\hline GS2 & .46 & - & & 1.72 & 0.84 & .60 \\
\hline GS3 & .39 & .41 & - & 1.37 & 0.89 & .58 \\
\hline GS4 & $.28 *$ & $.24^{*}$ & .36 & 1.14 & 0.99 & .68 \\
\hline \multicolumn{7}{|c|}{ Concentration } \\
\hline Con 1 & - & & & 2.35 & 0.69 & .57 \\
\hline Con2 & .37 & - & & 2.03 & 0.80 & .56 \\
\hline Con 3 & .39 & .37 & - & 2.02 & 0.69 & .57 \\
\hline Con 4 & $.24^{*}$ & .29 & $.25^{*}$ & 1.95 & 0.68 & .64 \\
\hline \multicolumn{7}{|c|}{ Freedom from Worry } \\
\hline Wor 1 & - & & & 1.26 & 0.96 & .61 \\
\hline Wor2 & $.29 *$ & - & & 1.52 & 0.94 & .52 \\
\hline Wor3 & .32 & .33 & - & 2.07 & 0.93 & .42 \\
\hline Wor4 & $.03 * *$ & $.25^{*}$ & .45 & 2.11 & 0.86 & .58 \\
\hline \multicolumn{7}{|c|}{ Confidence and Achievement Motivation } \\
\hline CM1 & - & & & 1.91 & 0.67 & .46 \\
\hline CM2 & .27 & - & & 2.52 & 0.64 & .47 \\
\hline CM3 & $.16^{* *}$ & .27 & - & 2.14 & 0.83 & .49 \\
\hline CM4 & .30 & $.16 * *$ & .23 & 1.87 & 0.79 & .47 \\
\hline \multicolumn{7}{|c|}{ Coachability } \\
\hline Coa1 & - & & & 2.29 & 0.90 & .65 \\
\hline Coa2 & $.18 * *$ & - & & 2.66 & 0.53 & .43 \\
\hline Coa3 & $.18 * *$ & .41 & - & 2.39 & 0.70 & .28 \\
\hline Coa4 & $.01 * *$ & .27 & .49 & 2.38 & 0.75 & .46 \\
\hline
\end{tabular}

Note. $1,2,3=$ order of the item in the subscale; $\alpha$ ? = Cronbach alpha of the subscale if item deleted.

*borderline correlation; **weak correlation.

scale exceeded .30 . Exceptions are the Coa1 $(.14$, low $)$ and Wor 1 (.29, borderline) items.

Coping with Adversity (Cop)

Based on the correlation, coefficient it can be stated that item 4 ("I am able to control my emotions regardless of the course of events.") has a weak correlation with other items. Generally, however, this subscale is relatively consistent.

Peaking under Pressure (Pea)

The item analysis suggests a strong correlation between the items. All items are suitable for subscale development. 


\section{Goal Settings/Mental Preparation (GS)}

The results suggest that the items are suitable for subscale development.

\section{Concentration (Con)}

The results suggest that the items are suitable for subscale development.

\section{Freedom from Worry (Wor)}

The correlation between items 1 and 4 is very weak (.03). The weak correlation is reflected in the borderline corrected correlation of item 1 with the subscale (.29). Other coefficients show sufficient values. Generally speaking, the items are suitable for subscale development.

\section{Confidence and Achievement Motivation (CM)}

In terms of item correlations, the subscale is problematic. Many values are low, especially CM4 with CM2 (.16) and $\mathrm{CM} 2$ with $\mathrm{CM} 3(.17)$. This suggests that the correlations between the items are rather weak. Nevertheless, the values of corrected item correlations suggest a medium correlation with the factor. Overall, however, the correlations are too weak to make a subscale out of four items with sufficient internal consistency.

\section{Coachability (Coa)}

The Coa1 item has a very weak correlation with other items in the subscale and shows a low corrected correlation (.14). Other items are sufficiently correlated for subscale development.

Based on the subscale item analysis, it is suggested that the Coal item be excluded. The remaining parts of the results section include both the 28-item model and the 27 -item model. An item analysis of the entire scale was performed. All corrected correlations between the items and the scale exceeded .25 except Wor1 (.08), Wor2 (.00), and Wor4 (.16). All three problematic items belong to the Freedom from Worry subscale.
Because of its weak (in one case even negative) correlation with other subscales (Table 3), its exclusion is considered in further analyses.

\section{Internal consistency of scales}

Table 4 shows the internal consistency of subscales and the entire scale of the Czech version compared with the original English version and Spanish version.

In the present study, values exceeding .70 were achieved only in Peaking under Pressure (.78). Five subscales show questionable, borderline values. These include Goal Setting/Mental Preparation (.68), Concentration (.65), adapted Coachability (.65; .53 in the case of retaining the Coal item), Freedom from Worry (.64), and Coping with Adversity (.64). The Confidence and Achievement Motivation subscale (0.55) displays poor reliability. Internal consistency of the entire questionnaire - Coping Resources - is good: .86, and .87 after exclusion of the Freedom from Worry subscale.

\section{Confirmatory factor analysis}

Figure 1 shows the Metric model and the structure of the Czech version of ACSI-28 with 27 items.

Table 5 shows the results of CFA for two models the Czech 28-item model and the Czech 27-item model (without Coa1 item - for more details, see the item analysis above).

The CFI and TLI values are borderline and probably reflect the weaker correlations between some items of individual subscales. The RMSEA and SRMR values are acceptable. Therefore, it can be stated that they confirm the theoretical model relatively well.

Seven of the 28 items had factor loadings of less than .50, with four of them being less than .30 (Figure 1). In general, items associated with the Freedom from Worry and Coachability factors exhibited the weakest loadings on the general factor.

A $2^{\text {nd }}$ order confirmatory factor analysis of the entire scale was performed (Table 5). The average scores of individual subscales that saturate the Coping

Table 3

Correlation matrix of the subscales

\begin{tabular}{lcccccc}
\hline Scale & 1 & 2 & 3 & 4 & 5 & 6 \\
\hline 1. Coping with Adversity & - & & & & & \\
2. Peaking under Pressure & .46 & - & & & & \\
3. Goal Setting/Preparation & .26 & .26 & - & & & \\
4. Concentration & .55 & .45 & .24 & - & & \\
5. Freedom from Worry & .28 & .14 & $-.17^{* *}$ & $.20^{* *}$ & - & \\
6. Confidence and Achievement Motivation & .37 & .41 & .48 & .45 & $.01^{* *}$ & - \\
7. Coachability & .26 & $.14^{* *}$ & $.18^{* *}$ & .35 & $.11^{* *}$ & .38 \\
\hline
\end{tabular}

**weak correlation. 
Table 4

Comparison of means, SDs, and Cronbach alpha of the Czech sample, the original sample in English and the Spanish sample

\begin{tabular}{|c|c|c|c|c|c|c|c|c|c|}
\hline \multirow[b]{2}{*}{ Scale } & \multicolumn{3}{|c|}{ Czech } & \multicolumn{3}{|c|}{ English } & \multicolumn{3}{|c|}{ Spanish } \\
\hline & $M$ & $S D$ & $\alpha$ & $M$ & $S D$ & $\alpha$ & $M$ & $S D$ & $\alpha$ \\
\hline Coping with Adversity & 7.04 & 2.29 & .64 & 6.26 & 2.23 & .66 & - & - & .57 \\
\hline Peaking Under Pressure & 6.99 & 2.79 & .78 & 6.40 & 2.87 & .78 & - & - & .70 \\
\hline Goal Setting/Mental Preparation & 5.60 & 2.57 & .68 & 5.48 & 2.75 & .71 & - & - & .65 \\
\hline Concentration & 8.34 & 2.00 & .65 & 7.02 & 2.19 & .62 & - & - & .52 \\
\hline Freedom from Worry & 6.96 & 2.49 & .62 & 6.46 & 2.82 & .76 & - & - & .71 \\
\hline Confidence and Achievement Motivation & 8.43 & 1.92 & .55 & 7.86 & 2.28 & .66 & - & - & .53 \\
\hline Coachability & 9.72 & 1.88 & $.53 / .65$ & 8.87 & 2.43 & .72 & - & - & .70 \\
\hline Total & 53.08 & 9.74 & $.83 / .85$ & 48.35 & 9.64 & .86 & - & - & .83 \\
\hline
\end{tabular}

Note. A dash indicates that the data were not reported. The values before and after a slash are the values before and after excluding one item from the Coachability subscale.

Table 5

Results of confirmatory factor analysis with fit indexes

\begin{tabular}{|c|c|c|c|c|c|c|c|}
\hline Model & $\chi^{2}$ & $d f$ & TLI & CFI & RMSEA & $90 \% \mathrm{CI}$ & SRMR \\
\hline \multicolumn{8}{|l|}{ First order factor analysis } \\
\hline Czech ACSI 28 item & 506 & 329 & .83 & .85 & .053 & {$[.044, .062]$} & .077 \\
\hline Czech ACSI 27 item & 458 & 303 & .85 & .87 & .052 & {$[.042, .061]$} & .075 \\
\hline Original ACSI (in English) & 875 & 329 & - & .91 & .044 & - & - \\
\hline Spanish ACSI & - & 329 & - & .91 & .032 & {$[.033, .039]$} & - \\
\hline \multicolumn{8}{|l|}{ Second order factor analysis } \\
\hline Czech ACSI 7 subscale & 65.8 & 14 & .75 & .83 & .135 & {$[.102, .160]$} & .073 \\
\hline Czech ACSI 6 subscale & 39.9 & 9 & .82 & .89 & .130 & {$[.900, .172]$} & .055 \\
\hline
\end{tabular}

Note. $d f=$ degrees of freedom; TLI = Tucker-Lewis index; CFI = comparative fit index; RMSEA = root mean square error of approximation; $\mathrm{CI}$ = confidence interval for RMSEA; SRMR = standardized root mean square residual. A dash indicates that the data were not reported.

Resources construct were used as manifest variables. Regarding the results of the item analysis, two models were considered - one with the Freedom from Worry subscale excluded. The model containing the Freedom from Worry subscale has a worse model-data agreement (Table 5), and the factor loading of this subscale is weak (.21). Figure 2 shows both the original 7-factor model and the proposed 6-factor model.

However, even after excluding the Freedom from Worry subscale, the model-data agreement is not satisfactory (high value of RMSEA $=.13$ ). The parallel analysis suggests that the number of factors in the data is three.

\section{Comparison of soccer referees by level}

Table 6 shows a comparison of referees based on their level of performance. Three subscales show significant differences between the groups. These are Peaking under Pressure ( $p=.03 ; d=0.37$, small difference), Concentration ( $p=.05 ; d=0.31$, small difference) and Confidence and Achievement Motivation ( $p=.01 ; d=0.41$, medium difference). The whole Coping Resources scale ( $p=.02$, $d=0.37)$ and Total 27-item Scale $(p<.01, d=0.41)$ also show small statistically significant differences.

\section{Discussion}

\section{Item analysis and reliability}

The vast majority of items appear suitable for the development of the subscales. However, this does not apply to item Coa1 in the Coachability subscale ("When a coach or manager tells me how to correct a mistake I've made, I tend to take it personally and feel upset."), which shows very weak correlations with other items and has a low corrected correlation with the subscale (.14). Regarding the fact that the subscale has only four items, its replacement or thorough reformulation should be considered in the future. For current purposes of practical application, it is recommended 
excluding this item. Therefore, the final Czech version of the questionnaire for soccer referees includes a total of 27 items.

The unsuitability of this item might be explained by the fact that the original questionnaire is primarily intended for athletes and not soccer referees. It is, therefore, possible that this specific population considers the items differently. This assumption would need

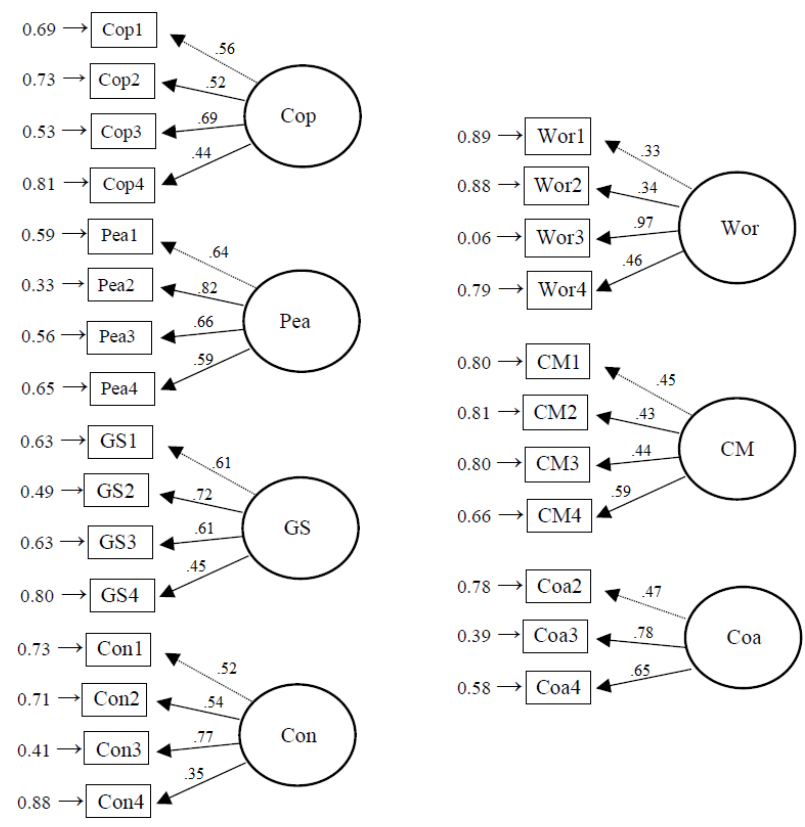

Figure 1. Metric model and the structure of the Czech version of ACSI-28 with 27 items. Cop = Coping with Adversity; Pea $=$ Peaking under Pressure; GS $=$ Goal Settings/Mental Preparation; Con $=$ Concentration; Wor $=$ Freedom from Worry; $\mathrm{CM}=$ Confidence and Achievement Motivation; $\mathrm{Coa}=$ Coachability. to be confirmed on a population of the Czech athletes in future research.

Taking into consideration the standard Cronbach's alpha value of 70 as the reliability borderline criterion (P. Kline, 1993), some subscales appear acceptably reliable (Peaking under Pressure .78) or display questionable reliability (Goal-Setting/Mental Preparation .68 , Concentration .65, Coachability .65 and .55 before exclusion of 1 item, Coping with Adversity .64, Freedom from Worry .62). Most of the alpha coefficients reported in the original standardization sample of the English version were similar to those of the present study (between .62 and .78 (Smith et al., 1995). The only subscale displaying poor reliability in the Czech adaptation is the Confidence and Achievement Motivation (.55 compared to the original .66). It suggests its very limited utilisation in applied practice.

More detailed analysis of Czech data implies that all items are relatively suitable for creating of Confidence and Achievement Motivation subscale (the values of corrected correlations in all items with the subscale are medium - higher than .30). On the other hand, the present items are not sufficiently correlated to each other to make an internally consistent subscale with only four items. The weak correlations of the items might be explained by the fact that this subscale, in principle, combines two different qualities and represents a vast construct. It can be assumed that selfconfidence and motivation qualities are correlated - for example, self-confidence and commitment, are considered by the Durand-Bush, Salmela, and GreenDemers (2001) to be part of one factor - the so-called fundamental skills.
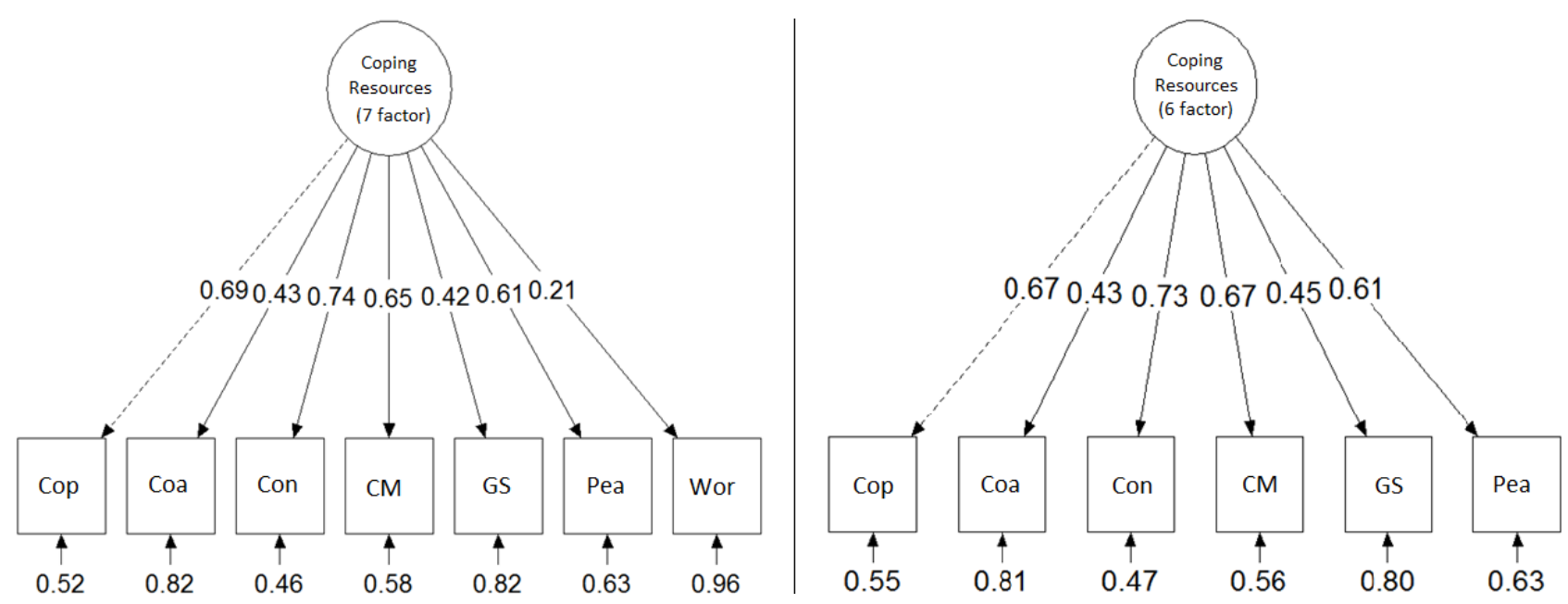

Figure 2. Original 7-factor model and the proposed 6-factor model. Cop = Coping with Adversity; Coa = Coachability; Con = Concentration; $\mathrm{CM}=$ Confidence and Achievement Motivation; GS = Goal Settings/Mental Preparation; Pea $=$ Peaking under Pressure; Wor $=$ Freedom from Worry . 
Table 6

Differences between national and regional Czech soccer referees

\begin{tabular}{|c|c|c|c|c|c|c|c|}
\hline \multirow[b]{2}{*}{ Topic } & \multicolumn{2}{|c|}{ (Inter)National } & \multicolumn{2}{|c|}{ Regional } & \multirow[b]{2}{*}{$t$} & \multirow[b]{2}{*}{$p$} & \multirow{2}{*}{$\begin{array}{c}\text { Cohen's } \\
d\end{array}$} \\
\hline & $M$ & $S D$ & $M$ & $S D$ & & & \\
\hline Coping with Adversity & 7.39 & 2.22 & 6.89 & 2.32 & 1.40 & .16 & 0.22 \\
\hline Peaking Under Pressure & 7.66 & 2.76 & 6.71 & 2.76 & 2.23 & .03 & 0.37 \\
\hline Goal Setting/Mental Preparation & 5.95 & 2.80 & 5.45 & 2.46 & 1.26 & .21 & 0.19 \\
\hline Concentration & 8.76 & 1.86 & 8.16 & 2.04 & 1.94 & .05 & 0.31 \\
\hline Freedom from Worry & 7.34 & 2.53 & 6.80 & 2.47 & 1.40 & .16 & 0.22 \\
\hline Confidence and Achievement Motivation & 8.98 & 1.94 & 8.20 & 1.86 & 2.67 & .01 & 0.41 \\
\hline Coachability ( 3 item) & 7.41 & 1.52 & 7.44 & 1.55 & -0.15 & .88 & 0.02 \\
\hline Coping Resources ( 23 item Czech ACSI 6 subscale) & 46.15 & 8.84 & 42.85 & 8.84 & 2.40 & .02 & 0.37 \\
\hline Total scale (27 item Czech ACSI 7 subscale model) & 53.49 & 9.33 & 49.66 & 9.43 & 2.63 & $<.01$ & 0.41 \\
\hline
\end{tabular}

Another explanation concerns a different understanding of the items in various socio-cultural environments. Confidence and Achievement Motivation subscale shows poor reliability in the Spanish translation, too (Sanz et al., 2011): The value of Cronbach's alpha is even lower than in the Czech sample (.53). This consistency may suggest more general psychometric issues concerning the validity of the subscale Confidence and Achievement Motivation connected with adapting this tool to different cultural settings. Supporting this presumption, different responses of Czech athletes to questions relating to mental skills of self-confidence and Commitment related to motivation in comparison with the Canadian sample are highlighted in a study by Vičar (2018).

It should be emphasised that during the development of the Confidence and Achievement Motivation subscale the Smith et al. (1995) chosen from a wide range of items based on their suitable correlation. However, in our Czech adaptation, we did not have a similarly extensive battery, as only the items in the latest version of the questionnaire ACSI-28 were translated. Future solutions include either extending the number of items in this subscale or its division into two subscales (titled e.g., Confidence and Achievement Motivation) and, again, increasing the number of items of each mental skill.

Globally, it is necessary to point out that a rather low level of internal consistency characterises not only many subscales from the Czech version, but also the original English and Spanish versions of the ACSI-28 questionnaire. On one hand, Loewenthal and Lewis, (2018) state that reliability coefficients higher than .60 can be deemed satisfactory provided that the following requisites are met: the scales are concise, and the validity of the construct is satisfactory. Yet, the inaccuracy of the measurement remains considerable both in the Czech adaptation and the original version. As the authors of the original questionnaire state their ambition to use the questionnaire for working with athletes in clinical practice (Sanz et al., 2011), we recommend adding some new correlating items to most of the subscales in favour of future development of the ACSI-28. Because the internal consistency is generally highly dependent on the number of items (Urbánek, Denglerová, \& Širůček, 2011) it possibly enables the subscales to be used separately for more valid measurement.

\section{Confirmatory factor analysis}

A lower level of internal consistency of some subscales is associated with borderline values of the model fit indexes. The proposed Czech 27-item alternative shows a better model fit than the 28 -item model. The value of the CFI of the questionnaire is .87 , and the TLI equals .85. It should be close to 1 , and a value of .90 is considered to be very good (R. B. Kline, 2015). On the other hand, RMSEA of the Czech version is .055 , and SRMR is .075. The upper value of the $90 \%$ confidence interval should not exceed .10 (R. B. Kline, 2015). Therefore, the results suggest that the model exhibited adequate fit comparable with the original, as original index values are also borderline.

The analysis of the questionnaire as one scale (referred by the authors to as Coping Resources) suggests that Freedom from Worry subscale should not be included in the total score for the population of the Czech referees. The following arguments support our recommendation. Firstly, three items of the Freedom from Worry subscale show a weak corrected correlation with the entire scale. Secondly, the correlation of this subscale with other subscales (.21) and the factor loading in relation to the overall scale (ranging from -.17 to .28) is low. Moreover, the internal consistency of the whole Coping Resources scale after excluding the Freedom from Worry subscale is slightly higher (.87 as opposed to .86 ). Finally, the $2^{\text {nd }}$ order 6 -factor model 
shows a significantly more acceptable model fit than the 7-factor.

Nevertheless, the results of the 6-factor CFA model without Freedom from Worry subscale are not completely satisfactory either - especially the RMSEA value is too high (.130). This suggests that the model is not sufficiently well specified. The reason may be that the Czech adaptation for referees is not a unidimensional scale. This assumption is supported by a parallel analysis of the Czech data, which estimated the number of three factors. The factor structure of the entire Coping Resources scale was not sufficiently explored in previous studies. The CFA values of model fit are not given in the articles. Consequently, we cannot state with certainty that this 3-factor division is not specific just for the Czech socio-cultural environment, or even more, for the population of Czech soccer referees. Therefore, the factor structure of the entire questionnaire should be subject to further studies.

The overall measure of the personal Coping Resources (23 items after excluding Freedom from Worry subscale and Coa 1 item from Coachability subscale) can be sufficient for certain applications in evaluating referees' psychological factors associated with their performance. Its good reliability $(0.87)$ meets the criteria for practical applications (Chráska, 2007). On the other hand, as alphas are highly influenced by the number of items in the scale, this result is more a function of the number of items than the greater validity of a single factor scale (as demonstrated in the models tested by CFA).

When suggesting the usefulness of the total score, the authors (Smith et al., 1995) consider that the correlations between the ACSI-28 subscales are quite low (between .10 and .55). Based on these findings, they believe that the subscales measure psychological skills that are quite distinct from one another. In other words, they presume that the general scale of personal Coping Resources constitutes a multifaceted coping skill construct (Carver, 1989). In the present research, the correlations between the subscales of the Czech version of the ACSI-28 are also quite low (between .14 and .55). The finding supports the conclusion that the total score and the scale reflect a multifaceted construct that can be used to assess sport-specific coping skills in referees.

\section{Criterion validity - identifying differences among competition levels}

The authors state that parts of the ACSI-28 are able to discriminate the level of athletes competing at different performance levels. In the original study (Smith et al., 1995), differences are found between high school athletes in Coachability, Concentration, and Coping with Adversity, and also in the total Coping Resources. In the Spanish study (Sanz et al., 2011), the differences discovered between athletes competing at a regional, national and international level were discovered in the subscales of Goal-Setting/Preparation, Confidence, and Achievement Motivation and also overall Coping Resources. Similar differences are visible in Czech soccer referees performing at different league levels. Referees judging matches at a national or international level report significantly higher quality of coping resources than those performing at a regional level. Moreover, the data shows significantly higher values in the subscales of Peaking under Pressure, Concentration and Confidence, and Achievement Motivation. It can, therefore, be stated that the results of the present study support the criterion validity of the Czech version of the questionnaire and 3 of its subscales.

\section{Recommendations and limitations}

For the further development of the ACSI-28 questionnaire, dividing Confidence and Motivation subscale into two unique subscales should be considered. Adding more items to all subscales could result in the greater reliability of the questionnaire, better clinical usability of separate subscales, and wider adaptation options into various languages, socio-cultural contexts, and populations. Finally, the $2^{\text {nd }}$ order factor structure of the overall scale should be subject to further studies, as the Czech data suggest the three-factor structure seems to be more accurate than the structure proposed in the original study.

The limitations of the present study are associated predominantly with the research sample. Firstly, it is rather small, yet representing a significant percentage of the total population of soccer referees in the Czech Republic. Secondly, since most soccer referees in the Czech Republic are male, it is difficult to judge whether there are any differences between men and women.

\section{Conclusions}

The Czech version of the Athletic Coping Skills Inventory ACSI-28 adapted for soccer referees (titled ACSI-27 for Soccer Referees) contains 27 items. The factor structure was assessed satisfactorily. The Coping Resources scale contains 23 items (excludes subscale Freedom from Worry) and provides a valid measurement, precise enough to be used in clinical practice. The reliability in the form of internal consistency is acceptable or borderline in most of the subscales, the subscale of Confidence and Achievement Motivation showed poor reliability. Therefore, practitioners should be aware of the limitations concerning the accuracy 
of measurement, while administrating individual subscales to soccer referees.

The results of our study open ways for wider application of the ACSI-28 questionnaire in the future. With further minor adaptations, administration to referees in various sports could be considered.

\section{Conflict of interest}

There were no conflicts of interest.

\section{References}

Anshel, M. H., \& Weinberg, R. S. (1995). Sources of acute stress in American and Australian basketball referees. Journal of Applied Sport Psychology, 7, 11-22.

Banville, D., Desrosiers, P., \& Genet-Volet, Y. (2000). Translating questionnaires and inventories using a cross-cultural translation technique. Journal of Teaching in Physical Education, 19, 374-387.

Carver, C. S. (1989). How should multifaceted constructs be tested? Issues illustrated by self-monitoring, attributional style and hardiness. Journal of Personality and Social Psychology, 56, 577-585.

Chráska, M. (2007). Metody pedagogického výzkumu [Pedagogical research methods]. Prague, Czech Republic: Grada Publishing.

Durand-Bush, N., Salmela, J. H., \& Green-Demers, I. (2001). The Ottawa Mental Skills Assessment Tool (OMSAT-3). Sport Psychologist, 15, 1-19.

Gencay, S. (2009). Magnitude of psychological stress reported by soccer referees. Social Behavior and Personality, 37, 865-868.

Goudas, M., Theodorakis, Y., \& Karamousalidis, G. (1998). Psychological skills in basketball: Preliminary study for development of a Greek form of the Athletic Coping Skills Inventory-28. Perceptual and Motor Skills, 86, 59-65.

Johansen, B. T., \& Haugen, T. (2013). Anxiety level and decision-making among Norwegian top-class soccer referees. International Journal of Sport and Exercise Psychology, 11, 215-226.

Kimbrough, S., DeBolt, L., \& Balkin, R.S. (2007). Use of the Athletic Coping Skills Inventory for Prediction of performance in collegiate baseball. Sport Journal, 10(1), 1-7.

Kline, P. (1993). The handbook of psychological testing. London, United Kingdom: Routledge.

Kline, R. B. (2015). Principles and practice of structural equation modeling (4th ed.). New York, NY: The Guilford Press.

Lesyk, J. J. (1998). The nine mental skills of successful athletes. Workshop presented at the Annual Conference of the Association for the Advancement of Applied Sport Psychology (AAASP). Hyannis, MA, USA.
Loewenthal, K., \& Lewis, C. A. (2018). An introduction to psychological tests and scales. London, United Kingdom: Psychology Press.

MacNamara, A., Button, A., \& Collins, D. (2010). The role of psychological characteristics in facilitating the pathway to elite performance. Part 1: Identifying mental skills and behaviours. Sport Psychologist, 24, 52-73.

Mathers, J. F., \& Brodie, K. (2011). Elite refereeing in professional soccer: A case study of mental skills support. Journal of Sport Psychology in Action, 2, 171-182.

Mellick, M. (2020). Soccer referee mental health: Developing a network of soccer referee mental health champions. In J. Dixon, J. B. Barker, R. C. Thelwell, \& I. Mitchell (Eds.), The psychology of soccer (pp. 278-291). New York, NY: Routledge.

Myers, N. D., Feltz, D. L., Guillén, F., \& Dithurbide, L. (2012). Development of, and initial validity evidence for, the Referee Self-Efficacy Scale: A multistudy report. Journal of Sport and Exercise Psychology, 34, 737-765.

Orlick, T. (1992). The psychology of personal excellence. Contemporary Thought on Performance Enhancement, 1, 109-122.

Salehi, H., \& Mohseni, M. (2018). Anxiety level among Iranian football referees: The role of self-consciousness and coping styles. Sports Management and Motor Behaviour Journal, 7(14), 16-25.

Samuel, R. D., Galily, Y., \& Tenenbaum, G. (2017). Who are you, ref? Defining the soccer referee's career using a change-based perspective. International Journal of Sport and Exercise Psychology, 15, 118-130.

Sanz, J. L. G., Pérez, L. M. R., Coll, V. G., \& Smith, R. E. (2011). Development and validation of a Spanish version of the Athletic Coping Skills Inventory, ACSI-28. Psicothema, 23, 495-502.

Smith, R. E., Schutz, R. W., Smoll, F. L., \& Ptacek, J. T. (1995). Development and validation of a multidimensional measure of sport specific psychological skills: The Athletic Coping Skills Inventory-2. Journal of Sport and Exercise Psychology, 17, 379-398.

Urbánek, T., Denglerová, D., \& Širůček, J. (2011). Psychometrika [Psychometrics]. Prague, Czech Republic: Portál.

Vallerand, R. J. (1989). Vers une méthodologie de validation trans-culturelle de questionnaires psychologiques: Implications pour la recherche en langue française [Toward a methodology for the transcultural validation of psychological questionnaires: Implications for research in the French language]. Canadian Psychology/Psychologie canadienne, 30, 662-680.

Vičar, M. (2018). Self-confidence, commitment and goalsetting in Czech athletes at different performance levels. Acta Gymnica, 48, 130-137.

Voborný, J., Zeman, T., Blahutková, M., \& Beňuš, R. (2015). Analysis of changes of mental state caused by psychological stress during match in football referees. Studia Sportiva, 9(1), 99-107. 